\title{
Automobile Lidar Sensorik: Stand, Trends und zukünftige Herausforderungen
}

\author{
M. Spies and H. Spies \\ Ingenieurbüro Spies, Hohenwart, Germany
}

Zusammenfassung. Lidar Sensorik gewinnt zunehmend an Bedeutung für die Realisierung und Verbesserung von aktuellen und zukünftigen Fahrerassistenzsystemen.

Seit die Halbleiterlasertechnologie für den Temperaturbereich in der Automobilanwendung verfügbar ist (ca. 1990), gab es immer wieder mehr oder weniger erfolgreiche Versuche, Lidar Sensorik im Fahrzeug einzusetzen. Erste Anwendung ist die adaptive Geschwindigkeitsregelung (ACC). Für diese Komfort-Funktionalität haben sich bisher jedoch Radarsensoren durchgesetzt.

Für Anwendungen, die den Fahrer wirklich entlasten und die Sicherheit erhöhen sollen, wie Spurwechselassistent, Stauassistent, Totwinkelüberwachung, Kreuzungsassistent und Precrashsensorik werden Sensoren benötigt, die bei großem horizontalen Erfassungsbereich (bis zu $180^{\circ}$ ) auch eine gute Winkelauflösung aufweisen, den Nahbereich $<20 \mathrm{~m}$ abdecken, eine hohe Datenwiederholrate haben und kostengünstig darstellbar sind. Für diese Anwendungen bietet sich die Lidar Sensorik an.

Im vorliegenden Vortrag werden die Grundlagen der Lidar Sensorik kurz erläutert, anhand von Beispielen verschiedene Ausführungsformen dargestellt sowie zukünftige Technologien und Anwendungsmöglichkeiten aufgezeigt.

\section{Physikalische Grundlagen}

\subsection{Messverfahren}

Mit Hilfe von Licht lässt sich auf viele Arten eine Entfernungsmessung darstellen. Für den automobilen Bereich müssen Entfernungen im Bereich 0 bis $200 \mathrm{~m}$ erfasst werden. Dafür bieten sich besonders Laufzeitverfahren an. Die gängigsten Verfahren sind in Fig. 1 dargestellt.

Correspondence to: M. Spies (martin.spies@ib-spies.de)
Beim ersten Verfahren wird die Phase des modulierten Lichtes ausgewertet. Der dafür nötige Hardware-Aufwand ist relativ gering. Das Verfahren ist aber nicht mehrzielfähig, Sichtweiteneinschränkungen führen zu einer "mittleren" Entfernung zwischen der Lage des Rückstreusignales der Sichtweiteneinschränkung und dem realen Ziel. Auch beim PMD (Photonic Mixer Device) um ein Modulationsverfahren, bei dem die Wägeschaltung (Phasenvergleich und Gleichlichtunterdrückung) in die Pixel integriert ist.

Bei den Impulsverfahren ergeben sich mehrere Möglichkeiten zur Auswertung der Lichtlaufzeit zwischen ausgesendetem und empfangenem Impuls: Mit dem Startsignal wird ein oder werden mehrere Zähler gestartet, der oder die mit Überschreitung einer Spannungsschwelle am Empfänger wieder gestoppt werden. Dieses Verfahren ist prinzipiell mehrzielfähig. Mit Zählerfrequenzen von $1,5 \mathrm{GHz}$ erreicht man eine Entfernungsauflösung von $10 \mathrm{~cm}$. Da die Vorverarbeitung bereits analog erfolgt, lässt sich die Impulsform des Rückstreusignals nicht auswerten und damit keine Aussage über die Art des Zieles mehr treffen.

Hierzu eignet sich das Verfahren der digitalen Signalerfassung, bei dem das Empfangssignal mit möglichst hoher Abtastrate (z.B. $150 \mathrm{MHz}$ entsprechend $1 \mathrm{~m}$ Lichtlaufzeit hin und zurück) digitalisiert wird.

Das digitale Rückstreusignal wird dann mit einem Mikroprozessor analysiert, es kann zwischen Sichtweiteneinschränkung und Ziel unterschieden werden.

\subsection{Rückstreuleistung}

Da man in der Automobilanwendung auch Ziele ohne Reflektor erkennen möchte um z.B. Schutzmaßnahmen einzuleiten, muss für das Objekt von einem diffusen Strahler mit einer Remission bis herunter zu 5\% ausgegangen werden. Solange nun die von dem Laser bestrahlte Fläche kleiner als die Objektfläche ist, sinkt die Energie am Empfänger proportional zu $1 / \mathrm{d}^{2} \mathrm{ab}$, ist die bestrahlte Fläche größer als das 

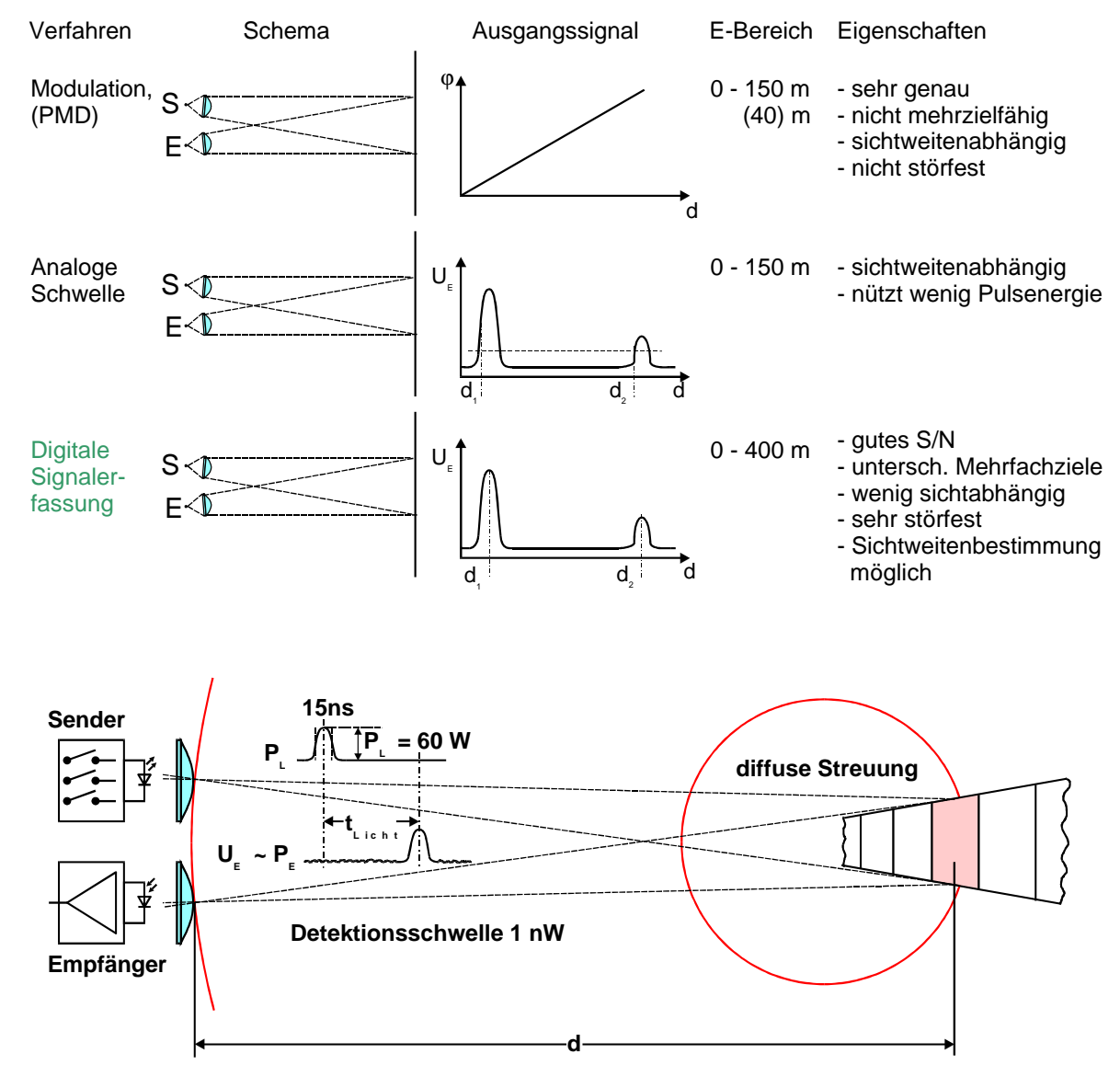

$P_{E}=k_{s y \text { siom }} \times P_{L} \times \frac{1}{d^{2}}$ für bestrahlte Fläche $F_{b \text { bostrant }}<$ Objektfläche $F_{\text {oblokt }}$

$P_{E}=k_{s y s t o m} \times P_{L} \times \frac{1}{d^{2}} \times \frac{F_{\text {obloke }}}{F_{\text {bostranll }}}$ für bestrahlte Fläche $F_{\text {bostran 11 }}>$ Objektfläche $F_{o \text { biokt }}$
Abbildung 1. Gängige optische Laufzeitverfahren.
Abbildung 2. Rückstreuleistung als Funktion der Entfernung.

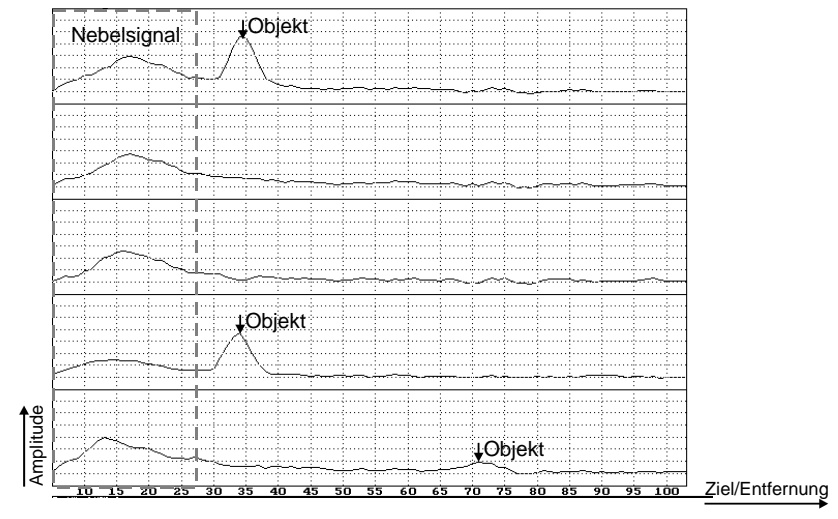

Abbildung 3. Rückstreusignal 5 Kanal System im Nebel, Sichtweite $\sim 100 \mathrm{~m}$.

Objekt erhält man eine Verringerung der Energie proportional $1 / \mathrm{d}^{4}$. Da durch die optische Abbildung des Strahles mit z.B. $1^{\circ} \times 2^{\circ}$ je Kanal nur kleine Flächen beleuchtet werden, gilt für ein normales Fahrzeug mit einer Fläche von 1,80 m Breite und 1,50 m Höhe die Proportionalität mit $1 / \mathrm{d}^{2}$ bis zu Entfernungen von $100 \mathrm{~m}$. Die Detektionsschwelle liegt bei der digitalen Signalerfassung bei ca. $1 \mathrm{nW}$ Lichtleistung am Empfänger. Durch Integration von mehreren Impulsen für eine Messung kann hierbei das Signalrauschverhältnis um die Wurzel der Anzahl dieser Integration erhöht werden. Figur 2 zeigt eine typische Anordnung bestehend aus Sender und Empfänger. Zur Verbesserung der Leistungsfähigkeit bei Sichtweiteneinschränkung sind die optischen Achsen von Sender und Empfänger getrennt. Dadurch ergibt sich ein Nahbereich eine geringe Überlappung der Sendeund Empfangsstrahlkegel und damit eine geringe Blendung des Systems bei Sichtweiteneinschränkung. Figur 3 zeigt Rückstreusignale eines 5-Kanal-Systems im Nebel bei einer Sichtweite von ca. $100 \mathrm{~m}$. Aus den unterschiedlichen Anstiegs- und Abfallzeiten zwischen "hartem" Ziel und Nebel und der festen Lage des Nebelsignals im Nahbereich des Systems können diese Signale unterschieden werden. 


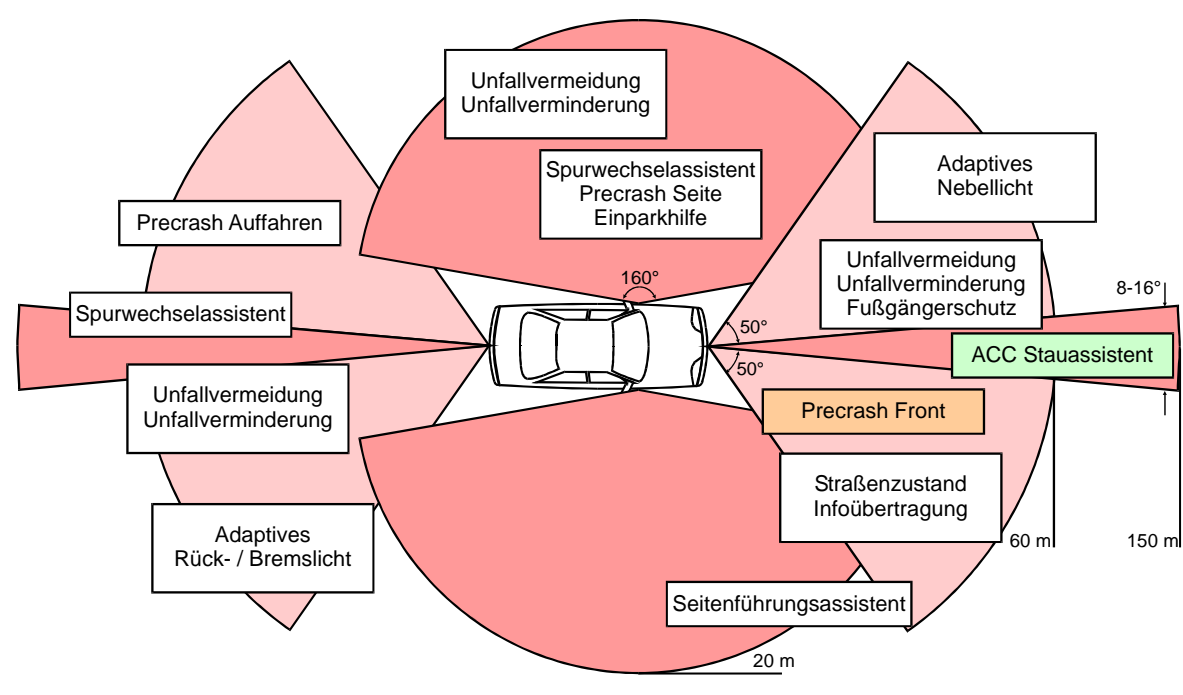

Abbildung 4. Anwendungsbereiche automobiler Lidar Sensorik.

\section{Automobile Lidar Sensorik}

\subsection{Erfassungsbereiche}

Zur Darstellung und Weiterentwicklung von Sicherheits- und Assistenzfunktionen im Kraftfahrzeug ergeben sich die in Fig. 4 dargestellten Erfassungsbereiche. Jeder dieser Bereiche besteht aus mehreren Kanälen, die entweder elektronisch oder mechanisch abgetastet werden und eine Information über die Lage und nach aufeinanderfolgender Beobachtung auch die Trajektorie eines Objektes liefern können. Wesentliche Daten sind die Entfernung, die Lage und die Relativgeschwindigkeit des Objektes bezogen auf das Fahrzeug.

\subsection{Realisierbare Sicherheits- und Assistenzfunktionen}

Ausgestattet mit dieser Sensorik lassen sich folgende Sicherheits- und Assistenzfunktionen realisieren: ACC Stop \& Go:

ACC Stop \& Go erweitert die Funktion des normalen aktiven Geschwindigkeitsreglers, der nur bei Geschwindigkeiten über $30 \mathrm{~km} / \mathrm{h}$ eingeschaltet ist und die eigene Geschwindigkeit an die der vorausfahrenden Fahrzeuge anpasst, um automatisches Anhalten und Wegfahren. Somit dehnt sich der Anwendungsbereich auch auf das Stadtgebiet aus. Der lästige Teil beim Fahren in der Kolonne, das Anhalten und Anfahren, wird automatisch erledigt.

Spurwechselassistent:

Fahrzeuge, die sich von hinten annähern, und Fahrzeuge auf den Nebenspuren werden beobachtet. Der Fahrer wird optisch oder haptisch vor einem Spurwechsel gewarnt, falls die Verkehrssituation diesen nicht zulässt.

Intelligente Heckleuchte:

Die Helligkeit der Heckleuchte wird an die Sichtbedingungen, an den Abstand und die Differenzgeschwindigkeit zum nachfolgenden Fahrzeug angepasst. Mit den gleichen Infor- mationen kann auch die Intensität des Bremslichts gesteuert werden.

Spurhaltungsassistent:

Vor allem in engen Baustellenbereichen auf Autobahnen wünscht man sich einen Assistenten, mit dessen Hilfe man zwischen der Mittelbarriere und einem LKW sicher navigieren kann. Die Information kann haptisch direkt auf das Lenkrad gegeben oder über eine Anzeige dargestellt werden.

Kreuzungsassistent:

In Verbindung mit GPS- und Kartendaten kann ein Kreuzungsassistent realisiert werden, der z.B. beim Überqueren oder Linksabbiegen auf eine vorfahrtsberechtigte Straße den Verkehr beobachtet und eine freie Lücke signalisiert.

Precrash-Sensorik:

Durch die Kenntnis der Richtung und der Geschwindigkeit des Unfallgegners in einer Unfallsituation können vor dem Aufprall Sicherheitsmaßnahmen getroffen werden. Dies sind z.B. reversible Gurtstraffer, Fußgängerschutz, aktive Passagierverlagerung. Auch die Airbag-Entfaltungszeiten werden durch die frühere Auslösung moderater. Damit könnte auf eine Sitzbelegungserkennung verzichtet werden. Eine unfallschwere- und richtungsabhängige Auslösung von Schutzmaßnahmen ist damit denkbar.

Lokale Datenübermittlung:

Die Infrarotsensorik eignet sich auch zur lokalen Datenübermittlung zwischen Fahrzeugen. So könnte z.B. der Bremsvorgang eines vorausfahrenden Fahrzeuges auch elektronisch an seinen Hintermann übermittelt werden. Im $\mathrm{Ge}$ gensatz zur Funkverbindung bleibt die Datenübermittlung auf die unmittelbare in Sichtweite befindlichen Verkehrsteilnehmer und z.B. aktive Verkehrszeichen beschränkt.

\subsection{Anforderungen an die Sensorsysteme}

Tabelle 1 zeigt die typischen Anforderungen an automobile Lidar-Systeme. 


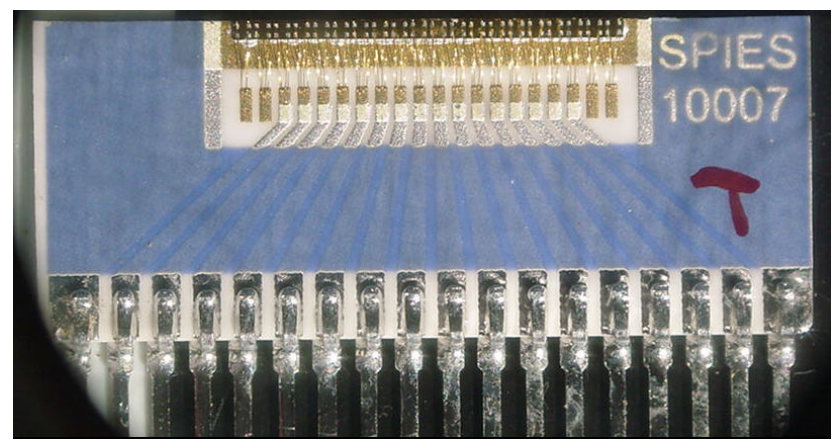

Abbildung 5. 20 Kanal Pulslaser Chip auf Substrat.

Tabelle 1. Anforderungen an Lidar-Systeme.

\begin{tabular}{ll}
\hline Reichweite (7\% Ziel) Fläche $2 \mathrm{~m}^{2}$ & bis 200 \\
Datenerneuerungsrate & $10-200 \mathrm{~ms}$ \\
Auflösung & $0.1 \mathrm{~m}$ \\
Genauigkeit & $\pm 0.5 \mathrm{~m}$ \\
Augensicher & Klasse 1 \\
& nach EN 60825-1 \\
vertikale Aufweitung in 1 bis 3 Ebenen & $2^{\circ}-5^{\circ}$ \\
horizontaler Erfassungsbereich mit & $10^{\circ}-180^{\circ}$ \\
$\begin{array}{l}\text { 16-32 Kanälen } \\
\text { geringer Einbauraum } \\
\text { leichte Integrierbarkeit }\end{array}$ \\
\hline
\end{tabular}

\subsection{Vergleich zwischen Lidar- und Radarsensorik}

Alle diese Funktionen sind auch mit Radarsensorik darstellbar. Tabelle 2 zeigt einen Vergleich zwischen Lidar- und Radarsensorik in, für die automobile Anwendung wichtigen Punkten.

Für die Lidar Sensorik sprechen im wesentlichen die geringeren Kosten, die bessere laterale Auflösung und die gute Korrelation zwischen Fahrersicht und Gerätesicht in Bezug auf reflektierende Markierung und Kennzeichnung von Straßen und Fahrzeugen sowie bezüglich Sichtweiteneinschränkungen durch Nebel oder Gischt.

\section{Schlüsselkomponenten}

Die Schlüsselkomponenten der Lidar Sensorik sind die Sende- und Empfangselemente. Für den kostengünstigen Aufbau von Mehrkanalsystemen wurden Singlechip Mehrfachlaser und Mehrfach Avalanche Photodioden entwickelt.

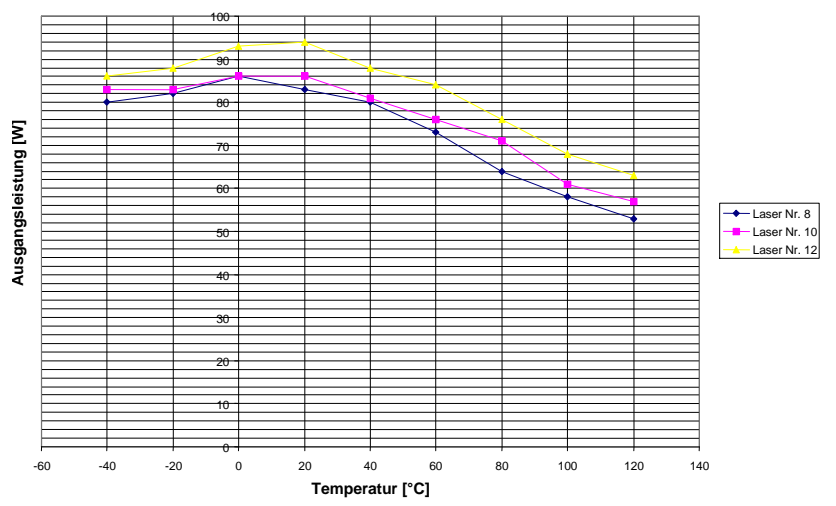

Abbildung 6. Ausgangsleistung als Funktion der Temperatur 20Kanal-Laser mit Ansteuerung.

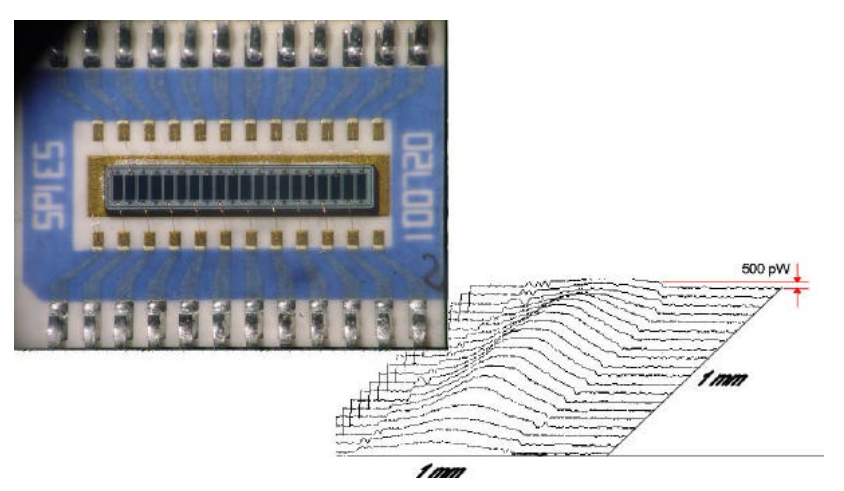

Abbildung 7.20 Kanal APD.

\subsection{Pulslaserdiodenarray}

Figur 5 zeigt ein Pulslaserdiodenarray mit 20 Laserdioden auf einem Dickschichtsubstrat. Die Pulsleistung jeder Diode beträgt zwischen $70 \mathrm{~W}$ und $85 \mathrm{~W}$ bei einer Impulslänge von ca. $16 \mathrm{~ns}$. Durch die MOCVD-Technologie ist die hohe Ausgangsleistung über einen weiten Temperaturbereich verfügbar und damit für die automobile Anwendung brauchbar. Figur 6 zeigt die Ausgangsleistung von 3 Dioden aus einem 16-Kanal Array mit der jeweiligen Ansteuerelektronik über den fahrzeugrelevanten Temperaturbereich von $-40^{\circ} \mathrm{C}$ bis $+120^{\circ} \mathrm{C}$.

\subsection{Photoempfängerarray}

Empfangsseitig wurde eine Avalanche Photodiodenarray entwickelt. Durch die innere Verstärkung von ca. 100-300 der Avalanchedioden erreicht man eine Verbesserung des Signalrauschverhältnisses um den Faktor 3-5 im Vergleich zu PIN-Dioden mit Vorverstärkern. Figur 7 zeigt ein 20-fach Singlechip Avalanche Photodiodenarray. Als 3-D-Graph ist die Empfindlichkeitsverteilung über die Fläche einer dieser Dioden $(0,5 \mathrm{~mm} \times 1 \mathrm{~mm})$ dargestellt. 
Tabelle 2. Vergleich Lidar - Radar.

\begin{tabular}{|c|c|c|}
\hline & Lidar & Radar \\
\hline Kosten relativ (ACC - System) & 1 & $\sim 2$ \\
\hline gängige Wellenlängen & $0.8-1 \mu \mathrm{m}$ & $4-12 \mathrm{~mm}$ \\
\hline Reichweite & bis $200 \mathrm{~m}$ & $\begin{array}{l}77 \mathrm{GHz} \text { bis } 200 \mathrm{~m} \\
24 \mathrm{GHz} \text { bis } 60 \mathrm{~m} \text { (Baugröße) }\end{array}$ \\
\hline laterale Auflösung & $\begin{array}{l}\text { gut darstellbar mit } \\
\text { Mehrkanalsensorik }<1^{\circ} \\
\text { (optische Abbildung der Strahlen) }\end{array}$ & $\begin{array}{l}\text { gut mit mechanisch gescannten oder phased array } \\
\text { Systemen (teuer); bei Mehrkanalsystemen nur } \\
\text { genaue Info über Radarschwerpunkt; } \\
\text { keine Korrelation zur Fahrzeugdimension }\end{array}$ \\
\hline Relativ-Geschwindigkeit & $\begin{array}{l}\text { muß über die zeitliche Ableitung } \\
\text { der Entfernungen berechnetwerden }\end{array}$ & direkt aus Doppelsignal \\
\hline Sichtweiteneinschränkung & $\begin{array}{l}\text { bestimmbar ist die physikalische } \\
\text { Rückstreuung des Mediums }\end{array}$ & $\begin{array}{l}\text { keine oder geringe Beeinflussung der Erlassungs - } \\
\text { leistung durch Nebel und Regen }\end{array}$ \\
\hline $\begin{array}{l}\text { einschränkende } \\
\text { Witterungsbedingungen }\end{array}$ & Nebel & starker Regen \\
\hline
\end{tabular}

$150 \mathrm{~m} / 15^{\circ}$

$16 \times 0,7^{\circ} / 0,2^{\circ}$ Abstand

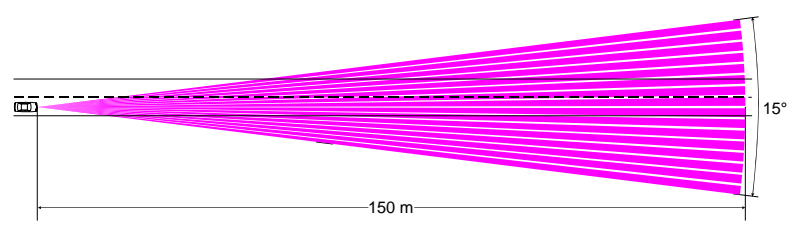

Abbildung 8. 16-Kanal-ACC-Sensor - Strahlgeometrie.

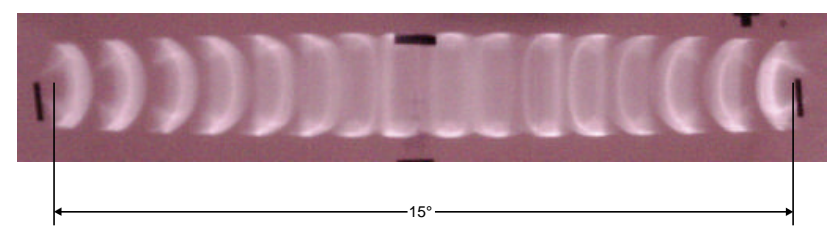

Abbildung 9. 16-Kanal ACC-Sensor - Laser-Abbildung.

\section{Anwendungsbeispiele}

\subsection{6-Kanal ACC-Sensor}

Zur Darstellung der ACC-Funktionalität (Adaptive Cruise Control) wurde ein 16-Kanal Sensor entwickelt und aufgebaut. Figur 8 zeigt die Strahlgeometrie dieses Sensors. Die reale Abbildung der Laser zeigt Fig. 9.

Das Blockschaltbild Fig. 10 zeigt die wesentlichen Komponenten dieses Sensors. Die gesamte Elektronik des Sensors ist in Fig. 11 dargestellt.

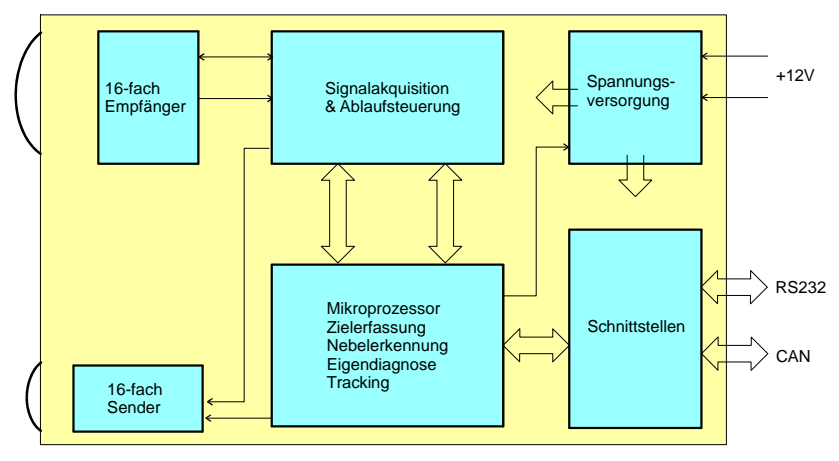

Abbildung 10. 16-Kanal ACC-Sensor - Blockschaltbild.

Einen Überblick über die Serienreifmachung und die jeweiligen Ausführungsformen gibt Fig. 12.

\subsection{Mechanisch abtastender Nahbereichssensor}

Für die Seitenführung und als Precrash-Sensor wurde ein Sensor entwickelt, der diese Funktionalität kostengünstig darstellt. Für die großen, zu erfassendenWinkelbereiche (siehe Fig. 13) bietet sich ein mechanisch abtastendes System an. Wie das Blockschaltbild Fig. 14 zeigt, werden bei diesem System nur die Optiken für Sender und Empfänger in einer kontinuierlichen Rotation bewegt.

Eine dynamische Anpassung der Winkelbereiche und Winkelauflösungen ist möglich. 


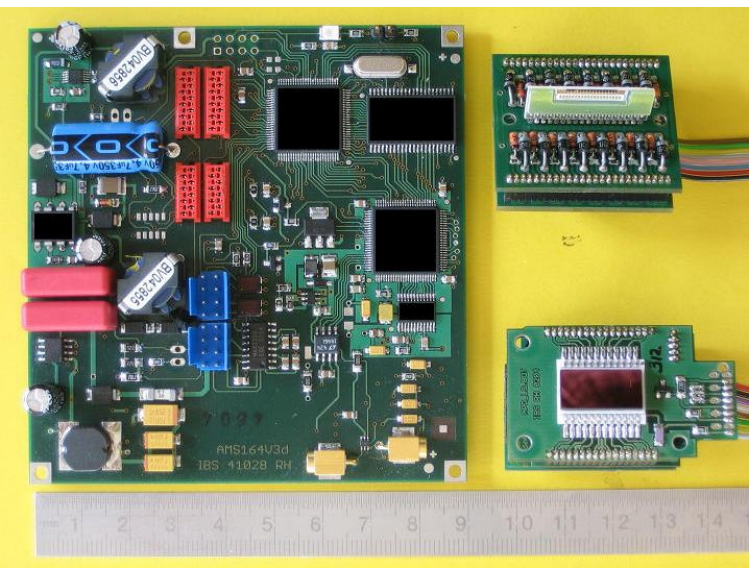

Abbildung 11. 16-Kanal ACC-Sensor-elektronische Komponenten.
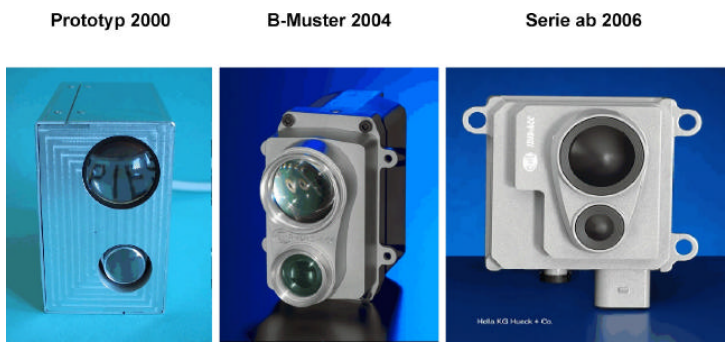

Abbildung 12. 16 Kanal-ACC-Sensor - vom Prototyp zum Seriengerät.

\section{Fazit und Ausblick}

Die Komponenten für die Lidar Sensorik im Automobil entsprechen den hohen Anforderungen und sind serienmäßig verfügbar.

Bisher durch Radarsensorik abgedeckte Funktion wie z.B. ACC lassen sich auch durch Lidar Sensorik darstellen und um wichtige Funktionen wie z.B. ACC Stop \& Go erweitern. Die bessere laterale Auflösung erweist sich für die Erkennung der eigenen Fahrspur als hilfreich. Die Möglichkeit der Sichtweitenbestimmung wiegt den Nachteil der verringerten Leistungsfähigkeit in Nebel und Gischt auf und bildet eine bessere Korrelation zwischen Geräte- und Fahrersicht. Mit Rücksicht auf andere Verkehrsteilnehmer sind "Blindfahrgeräte" schon aus Produkthaftungsgründen nicht erwünscht.

Lidar Sensorik ermöglicht echte Fahrerassistenz außerhalb des Fahrspaßes durch Unterstützung beim Wiederanfahren und Anhalten im Stau (ACC Stop \& Go), bei der Seitenführung und beim Spurwechsel, dann also, wenn das Fahren sonst zur Mühe wird.

Der Einsatz von Lidar Sensorik erlaubt eine Verbesserung der Reaktion von Rückhaltesystemen. Reversibel ansteuerbare Gurtstraffer, Airbags und sonstige passive Schutzmaßnahmen sind möglich.

Zukünftige flächige Laser- und Detektorarrays können in Verbindung mit Bildsensoren zur Unfallvermeidung beitra-

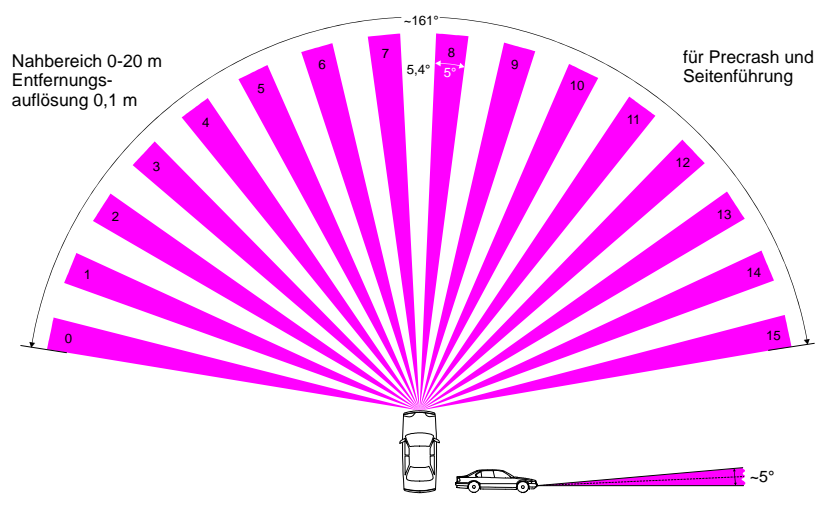

Abbildung 13. Mechanisch gescannter Nahbereichsensor - Strahlgeometrie.

Sensorkopf

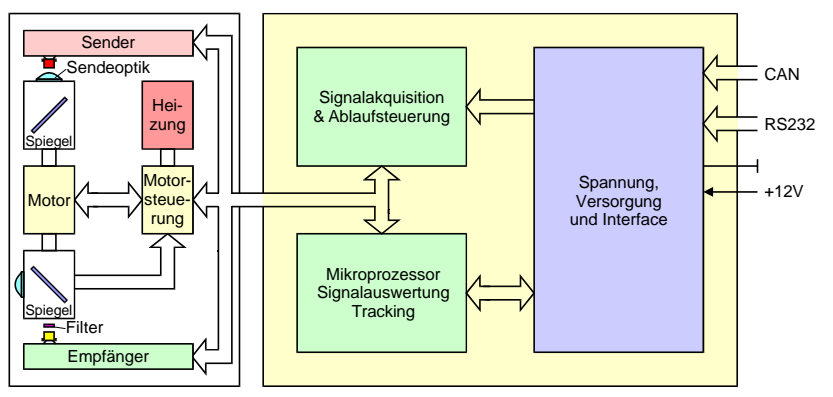

Abbildung 14. Mechanisch gescannter Nahbereichssensor - Blockschaltbild.

gen. Automatisches Notbremsen und Ausweichen mit Erkennung des freien Weges werden denkbar.

Für die sicherheitsrelevante Sensorik ist eine Sichtweitenabhängigkeit unerwünscht. Mit zukünftiger Technologie können nach dem hier vorgestellten Prinzip arbeitende Sensoren im Wellenlängenbereich von $6 \mu \mathrm{m}-10 \mu \mathrm{m}$ aufgebaut werden, die diese Einschränkung nicht mehr aufweisen.

\section{Literatur}

Infrared Components, Brochure No. 67CM, Santa Barbara Research Center, Goleta, ca. 1967.

Krüger, H.-P.: Fahrerassistenz von morgen - Anforderungen und Ausblick Kooperationsforum "Fahrerassistenz",Bayern innovativ, Aschaffenburg, 2005.

Spies, H. und Spies, M.: Sensor Systems - What is achievable today and in the near future $6^{\text {th }}$ Intern. Symposium and Exhibition on Sophisticated Car Occupant Safety Systems, Karlsruhe, Germany, 2002.

Spies, H. und Spies, M.: IR-Abstandssensoren zur Unfallvermeidung und -minderung Halbleiterlichtquellen, OTTI-Profiforum, Regensburg, 2004.

Spies, M.: IR-Multichannel Distance and Visibility Measurement, Conf. Microsystem Key to Automotive Safety Concepts, VDI / VDE-IT, Berlin, 1995. 